\title{
Conferring antioxidant capacity to cellulose based materials by using enzymatically-modified products
}

\author{
Oriol Cusola, Cristina Valls, Teresa Vidal and M. Blanca Roncero* \\ CELBIOTECH_Paper Engineering Research Group, Universitat Politècnica de Catalunya. \\ BarcelonaTech, Colom 11, E-08222 Terrassa, Spain. \\ * Corresponding author: roncero@etp.upc.edu; Tel.: +34-93 739-82-10; Fax: +34-93-739-81-01.
}

\begin{abstract}
A new, industrially feasible method for conferring cellulosic substrates antioxidant properties by using enzymatic products was developed. The method allows cellulose surfaces such as those of paper sheets (PS) to be conferred antioxidant capacity by using a functionalization solution (FS) obtained from an enzymatic reaction. Various laccases and different phenolic compounds $(\mathrm{PhC})$ potentially possessing antioxidant action were used to prepare the functionalization solutions. Antioxidant capacity was assessed by using UV spectrophotometry to monitor the inhibition of ABTS radical cation $\left(\mathrm{ABTS}^{{ }^{++}}\right.$) in the presence of antioxidants. Based on the results, enzymatic modification of the phenolic compound in the functionalization solution increases the bonding strength of its components to cellulosic materials. Evidences on the grafting of the FS onto the cellulosic sheets, and changes in the chemical structure of the resulting oxidized form of PhCs were assessed by using ATR-FTIR. The level of antioxidant capacity achieved depends on the enzyme type, the chemical structure of the compounds in FS, and the presence of lignosulfonates (SL) in it. The potential of the proposed method for conferring antioxidant properties to cellulose-based materials by surface application of a product obtained from an enzymatic reaction is demonstrated here for the first time.
\end{abstract}

Keywords: Laccase; antioxidant capacity; cellulose; phenolic compounds; $\mathrm{ABTS}^{\circ+}$
Abbreviations:
ABTS 2,2'-azino-bis(3-ethylbenzothiazoline-6-sulphonic acid)
Fb Cerrena Unicolor enzyme supplied by Fungal Bioproducts ${ }^{\circledR}$
FS Functionalization solution containing Lacc, PhC, and SL
FSU Functionalization solution uptake
KFS Control FS containing one or two of the additives Lacc, LG, SL in various combinations
Lacc Laccase
LG Lauryl gallate, dodecyl 3,4,5-trihydroxybenzoate
Mt Myceliphthora Thermophila enzyme
PhC Phenolic compound
PS Paper sheets
SL Sulfonated lignin
TEAC Trolox equivalent antioxidant capacity
Tv Trametes Villosa enzyme 


\section{Introduction}

The search for renewable, sustainable alternatives to petroleum-derived materials has gained interest in recent years. Cellulose can be an effective choice here by virtue of its natural abundance, renewable nature and unique properties. Therefore, special attention has been focused on the search for new and more valuable applications for cellulose (Qiu and Hu 2013; Morgado et al. 2013; Ma et al. 2013). The development of innovative materials with new or enhanced properties for use in the packaging industry has also aroused much interest lately, particularly as regards active packaging materials. Active packaging is an innovative concept that can be defined as a type of packaging that extends the shelf-life of foods while maintaining their safety and quality (Rudra et al. 2013; GómezEstaca et al. 2014; Kodal Coşkun et al. 2014) which is currently a central concern for the food industry. Antioxidant capacity is one of the most desirable properties for a material used to manufacture food-containing packaging. In fact, the quality and shelf-life of packed food are known to be diminished by the action of oxygen causing color and flavor alterations, lipid and fat oxidation and microbiological spoilage. Accordingly, the most common approaches to preventing food oxidation involve creating oxygen barriers by using glass, metal or plastics in combination with oxygen scavengers in packaging materials (López-de-Dicastillo et al. 2012; Raheem 2013). However, the reagents used in oxygen scavenger systems can migrate off the packaging or be channeled to the environment after use.

An antioxidant compound can be defined as a substance whose action can inhibit oxidation rate of a free radical. Oxidation reactions can in fact produce free radicals and the radicals start chain reactions that are deleterious to cells. Antioxidants terminate such reactions by removing free radical intermediates and inhibit other oxidation reactions by oxidizing themselves. Traditionally, food producers have prevented oxidation reactions by using synthetic antioxidants; however, their direct addition to foods limits their activity owing to their lack of selectivity to target the food surface where most oxidative reactions occur. This has aroused considerable interest, promoted by consumer's demands, in using non-detrimental antioxidants and reducing their presence in food.

The most common method for obtaining packaging materials with antioxidant properties involves incorporating appropriate natural antioxidants into plastic materials and assessing the risk of their migrating off the polymer in contact with food. In this context, López-de-Dicastillo et al. (López de Dicastillo et al. 2012), studied antioxidant packaging films consisting of ethylene vinyl alcohol (EVOH) copolymers, Barbosa-Pereira et al. (Barbosa-Pereira et al. 2013), assessed tocopherolcontaining active packaging films made of low density polyethylene (LDPE) and Kanatt et al. (Kanatt et al. 2012), investigated active chitosan-polyvinyl alcohol films with natural mint and pomegranate peel extracts. Cellulose derivatives such as microcrystalline, carboxymethyl and ethyl cellulose have previously been used as carrier materials to prepare advanced materials with antioxidant properties (Jansen et al. 2011; Hsu and Kilmartin 2012; Serrano Cruz et al. 2013); by contrast, paper has seemingly never to date been used as carrier material for this purpose.

The use of enzymes in technological processes has provided an environmentally friendly, costeffective alternative to the traditional chemicals used in industrial processes. Specifically, laccasemediator systems have brought about especially interesting advances in the pulp and paper industry, 
and allowed lignocellulosic materials with improved properties to be developed (Gao and Cranston 2008; Aracri et al 2011; Saastamoinen et al 2012; Valls and Roncero 2012; Aracri and Vidal 2012; Shogren and Biswas 2013). One interesting application of laccase systems exploits their ability to graft low-molecular weight phenolic compounds onto lignocellulosic materials (Aracri et al. 2010; Fillat et al. 2012). Phenolic compounds have also been reported to possess multiple biological effects, including antioxidant activity (Rice Evans et al. 1997; Robards et al. 1999; Kähkönen et al. 1999; Spizzirri et al. 2009). They represent a good alternative to substitute synthetic antioxidants, such as butylated hydroxytoluene (BHT), butylated hydroxyanisole (BHA) and tert-butylhydroquinone (TBHQ), which use as food additives is being questioned (López-de-Dicastillo et al. 2010; Tian et al. 2012).

In this work, we developed a methodology for applying a functionalization solution (FS) obtained by enzymatic reaction to the surface of previously formed paper materials. A similar method has already been reported regarding the hydrophobization of such materials (Cusola et al. 2012; Cusola et al. 2013; Cusola et al. 2014), but never before for conferring antioxidant properties. The main objective of the treatment was to obtain cellulose-based materials with antioxidant properties useful for packaging applications.

\section{Materials and methods}

\section{Paper, enzyme and chemicals}

Handsheets made from Eucalyptus ECF fibers (supplied by ENCE, Spain), and Filter paper sheets from FILTERLAB ${ }^{\circledR}$ (Sant Pere de Riudebitlles, Barcelona, Spain) were used for functionalization. The enzymes used were a laccase from Trametes Villosa (Tv) with an activity of $588 \mathrm{U} / \mathrm{mL}$, a laccase from Myceliphthora Thermophila (Mt) with an activity of $532 \mathrm{U} / \mathrm{mL}$ both supplied by Novozymes ${ }^{\circledR}$ (Bagsvaerd, Denmark), and a laccase from Cerrena Unicolor supplied by Fungal Bioproducts ${ }^{\circledR}$ (Fb) with an activity of $1660 \mathrm{U} / \mathrm{mL}$. Potassium persulfate (di-potassium peroxdisulfate), 2,2'-azino-bis(3ethylbenzthiazoline-6-sulphonic acid) (ABTS), 6-hydroxy-2,5,7,8-tetramethylchroman-2-carboxylic acid (trolox) and ethanol were obtained from Sigma-Aldrich. Twenty-eight synthetic and natural phenolic compounds $(\mathrm{PhC})$ potentially possessing antioxidant capacity were used in the laccase treatments, namely: caffeic acid (CA), para-hydroxybenzoic acid (pHB), acetosyringone (AS), syringaldehyde (SA), p-coumaric acid (pCA), vanillin (V), acetovanillone (AV), ferulic acid (FA), coniferyl aldehyde (CFA), sinapic acid (SPA), sinapyl aldehyde (SAD), violuric acid (VA), methyl syringate (MS), gallic acid (GA), methyl gallate (MG), ethyl gallate (EG), propyl gallate (PG), butyl gallate (BG) octyl gallate (OG), lauryl gallate (LG), stearyl gallate (SG), $\beta$-sitosterol ( $\beta$-S), $\alpha$ tocopherol ( $\alpha$-TP), 4-[4-(trifluoromethyl)phenoxy]phenol (TPH), isoamyl salicylate (IS), 2,4,6-tris(1phenylethyl)phenol (TRP), tannic acid (TA) and resveratrol (RV), all supplied by Sigma-Aldrich. Soluble sulfonated kraft lignin (SL) was obtained from Borreegard (Sarpsborg, Norway), and used as received. 


\section{Preparation of the functionalization solutions (FS)}

The treatment conditions were based on those reported in previous studies (Cusola et al. 2013; Cusola et al. 2014). The enzymatic treatments were performed in an agitated bath at $50^{\circ} \mathrm{C}$, under moderate agitation, using $250 \mathrm{~mL}$ beakers with final concentrations equivalent to $50 \mathrm{mM}$ sodium tartrate buffer $(\mathrm{pH} 4), \mathrm{PhC}(1.2 \mathrm{~g} / \mathrm{L})$, lignosulphonate $(1.2 \mathrm{~g} / \mathrm{L})$ and $1.2 \mathrm{U} / \mathrm{mL}$ laccase. The reaction was stopped by quenching the beakers with cold water. Then, the obtained solution (functionalization solution, FS) was applied to the surface of paper sheets by dipping. Several control treatments (control functionalization solutions, KFS), were also performed (Table 1) and the results were compared to the paper sheets before the surface treatments.

Table 1 Conditions to obtain the aqueous FS and controls (KFS) solutions used in the surface treatments of paper sheets. The control treatments consisted in several combinations of the compounds (PhC, SL and Lacc) present in the FS.

\begin{tabular}{lllll}
\hline & System & $\begin{array}{l}\text { Phenolic compound } \\
\text { dose }(\mathbf{g} / \mathbf{L})\end{array}$ & $\begin{array}{l}\text { Sulfonated lignin } \\
\text { dose }(\mathbf{g} / \mathbf{L})\end{array}$ & $\begin{array}{l}\text { Laccase dose } \\
(\mathbf{U} / \mathbf{m L})\end{array}$ \\
\hline FS & PhC+SL+Lacc & 1.2 & 1.2 & 1.2 \\
\hline \multirow{4}{*}{ KFS } & PhC & 1.2 & - & - \\
& SL & - & 1.2 & - \\
& Lacc & - & - & 1.2 \\
& PhC+Lacc & 1.2 & - & 1.2 \\
& SL+Lacc & - & 1.2 & 1.2 \\
\hline
\end{tabular}

\section{Paper sheets (PS) treatment with the FS and KFS}

Eucalyptus fibres above described were used to prepare handsheets according to ISO 5269-2:2004 on a Rapid-Köten lab former. Filter paper was used as received. Samples $\left(4 \mathrm{~cm}^{2}\right)$ of both paper substrates (handsheets and filter paper sheets) were cut off and soaked in each one of the FS or KFS derived from the Laccase-PhC enzymatic reactions by keeping the sheet submerged for 3 seconds. Then the paper sheet was removed, spread onto blotting paper, and allowed to dry in a normalized atmosphere $\left(23^{\circ} \mathrm{C}, 50 \% \mathrm{RH}\right)$.

In the case that the paper sheets were subjected to washing, this was performed by immersing the sheets in distilled water at $80^{\circ} \mathrm{C}$ for 30 min under constant agitation. Afterwards the sheets were also spread onto blotting paper, and dried in a normalized atmosphere $\left(23^{\circ} \mathrm{C}, 50 \% \mathrm{RH}\right)$.

In the case that the paper sheets were subjected to curing treatments, this was conducted by ovencuring at $150^{\circ} \mathrm{C}$ for $30 \mathrm{~min}$. Afterwards, the sheets were allowed to cool down under normalized atmosphere for moisture stabilization.

\section{Antioxidant capacity of the functionalization solutions (FS), and the pure phenolic compounds}

The antioxidant activity of the enzymatic systems (FS and KFS) was assessed by a procedure consisting in the quantification of the $\mathrm{ABTS}^{\circ+}$ radical decoloration described by several authors $(\operatorname{Re} e t$ al. 1999; Kubo et al. 2000). The decoloration is due to the interaction of this radical with hydrogen or 
electron donor species. The method consists in the addition of the antioxidant compound to a preformed $\mathrm{ABTS}^{\bullet+}$ radical solution and quantifying the remaining $\mathrm{ABTS}^{{ }^{+}}$after a fixed time period, by means of UV spectrophotometry (Fig. 1). The reduction in $\mathrm{ABTS}^{\circ+}$ concentration, induced by a certain concentration of antioxidant, is related to that of trolox and gives the TEAC (trolox equivalent antioxidant activity) value of that antioxidant (Arts et al. 2004). Experiments were performed on a Thermo Scientific Evolution 600 spectrophotometer. The absorbance value of each solution at $752 \mathrm{~nm}$ was monitored every minute up to 6 minutes, or until stabilization. The inhibition $\%$ was calculated as follows:

$$
\text { [inhibition \% } \left.=\left(A_{i}-A_{f}\right) / A_{i} \times 100\right]
$$

where $\mathrm{A}_{\mathrm{i}}$ is the initial $\mathrm{ABTS}^{\circ+}$ absorbance value, and $\mathrm{A}_{\mathrm{f}}$ is the $\mathrm{ABTS}^{\circ+}$ absorbance value after contact with the antioxidant compound aliquot.

The inhibition $\%$ was plotted against the concentration, and the slope of the curve was compared to that of trolox to calculate the TEAC. The TEAC was obtained from the ratio between the slope of the curve of the studied compound and the slope of the curve obtained for trolox.

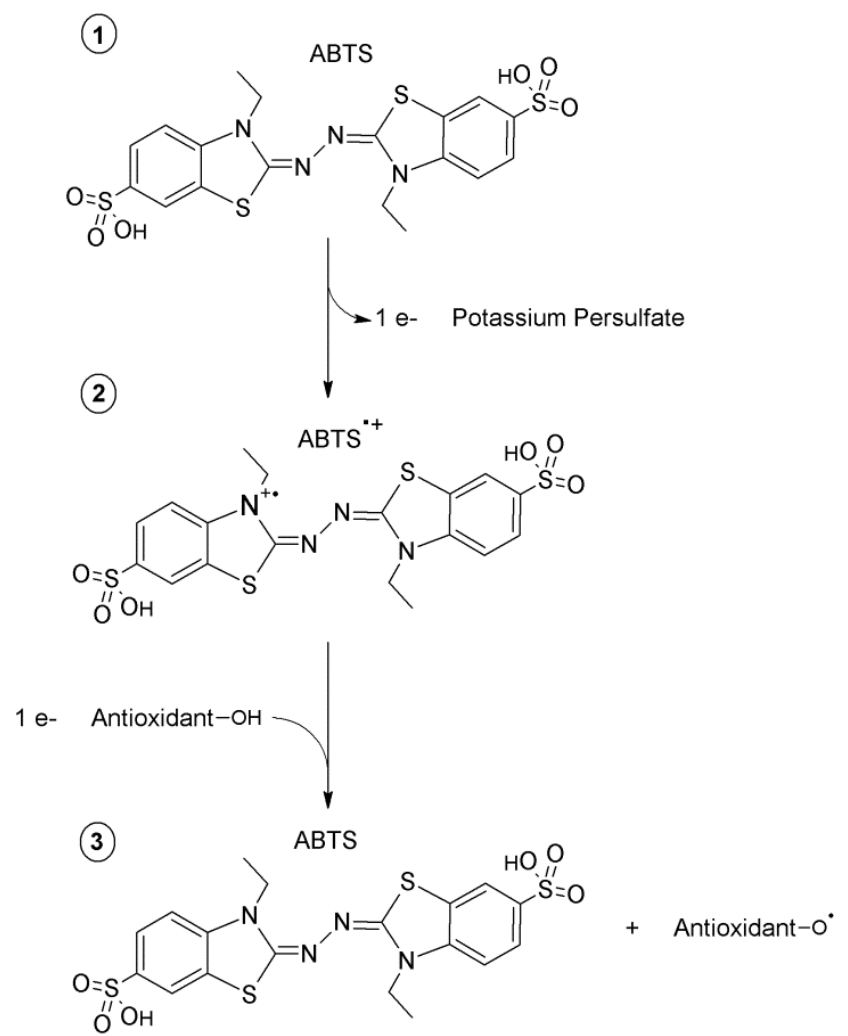

Fig. 1 Generation of the $\mathrm{ABTS}^{\cdot+}$ radical cation by reacting potassium persulfate with the ABTS salt (step 1-2), and reduction by an antioxidant substance (step 2-3). 


\section{Antioxidant capacity of treated papers}

The method to evaluate the antioxidant capacity of the treated papers was also based on the method developed by Serpen et al. (Serpen et al. 2007), for the measurement of the antioxidant activity of insoluble food components, by quantification of the $\mathrm{ABTS}^{\circ+}$ radical inhibition.

For the study of the antioxidant capacity of the treated papers, samples of $10 \mathrm{mg}$ of the treated paper sheets were cut in small pieces, weighed and introduced into microcentrifuge tubes. A volume of 1.7 $\mathrm{mL}$ of the $\mathrm{ABTS}^{\circ+}$ solution was added to each one of the microcentrifuge tubes. Next, the tubes were agitated using a vortex mixer for 2 minutes to facilitate the surface reaction, centrifuged for 4 minutes at $6000 \mathrm{rpm}$, and allowed to stand for 30 minutes before conducting the absorbance tests. The absorbance measures at $752 \mathrm{~nm}$ of the centrifuged tubes were performed by taking $1 \mathrm{~mL}$ of the supernatant part of the tubes and placing it into a methacrylate cubette. The absorbance value of each sample at $752 \mathrm{~nm}$ was monitored every minute up to 6 minutes, or until stabilization. The inhibition \% is calculated as indicated in equation 1 , however in this case (paper samples) the $A_{i}$ is the initial $\mathrm{ABTS}^{\circ+}$ absorbance value, and $\mathrm{A}_{\mathrm{f}}$ is the $\mathrm{ABTS}^{\circ+}$ absorbance value after contact with the paper samples.

\section{FTIR analysis}

ATR-FTIR spectra of paper sheets were recorded on a Perkin Elmer Spectrum 100 FT-IR spectrometer equipped with universal ATR sampling accessory. The spectra wavenumber range was $4000-550 \mathrm{~cm}^{-1}$ and the number of scans was 32, with a wavenumber resolution of $1 \mathrm{~cm}^{-1}$. Paper samples were placed directly on the ATR accessory and the measuring cell was washed using deionized water and ethanol between measurements.

For the FTIR analysis of the enzyme-modified LG, $200 \mathrm{~mL}$ of the FS corresponding to the LG compound were prepared as explained previously. Then, the solution was centrifuged at $6000 \mathrm{rpm}$ during $90 \mathrm{~min}$, and the supernatant eliminated from the sample. Afterwards, the sample was allowed to dry at room temperature until a dry solid residue was obtained, and placed into a desiccator overnight. Finally, it was finely grinded and placed directly on the ATR accessory.

\section{Results and Discussion}

\section{Screening of antioxidant capacity in PS upon treatment with the functionalization solution (FS)}

In a preliminary study we assessed the antioxidant capacity of the pure phenolic compounds, and their TEAC value (viz. the trolox equivalent antioxidant capacity) was reported (see Table 2, and Fig. 2). Results evidenced that several of the studied compounds presented antioxidant capacity, but others did not show such property at all. According to several authors (Robards et al. 1999; Nugroho Prasetyo et al. 2010), the antioxidant capacity of a given compound increases with the increasing - $\mathrm{OH}$ functions in the aromatic ring. This can be observed by comparing pHB and GA compounds, which presented the same hydroxybenzoic acid structure with the only difference that GA possess 2 additional $-\mathrm{OH}$ functions located at the meta positions. Additionally, other authors (Rice Evans et al. 1997) suggested that orto and para-substituted $-\mathrm{OH}$ functions do not provide antioxidant capacity, while the metasubstituted do. This may be the reason why the IS compound did not show antioxidant capacity. 
As it is shown in Fig. 2, compounds with a methoxy in the -meta position (V, AV, AS, SA, MS) showed higher antioxidant capacities than if they had had no substitution, but lower than if they had had -OH functions in such position. This observation is in accordance with already reported studies (Rice Evans et al. 1997; Nugroho Prasetyo et al. 2010). In a general way, it was observed that hydroxycinnamic acid structures presented higher antioxidant capacities than those corresponding to hydroxybenzoic acid structures.

Once the TEAC values of the pure compounds were measured and analyzed, the FS corresponding to each phenolic compound were obtained and applied to the surface of paper substrates. Then, the antioxidant capacity of the treated papers was assessed. Preliminary control tests were used to measure the antioxidant capacity of the eucalyptus handsheets (PS) and the enzyme (PS+lacc). These substrates exhibited 5\% and 7\% inhibition, respectively, so the controls possessed a low antioxidant capacity. Fig. 2 shows the antioxidant capacity of paper sheets treated with the functionalization solutions obtained by using the 25 phenol compounds. All enzymatic solutions increased that of the treated PS. This was particularly so with the functionalization solutions prepared from ferulic acid (FA), octyl gallate (OG), lauryl gallate (LG), 2,4,6-tris(1-phenylethyl)phenol (TRP) and resveratrol (RV). As can be seen from the TEAC values for the pure compounds, also shown in Fig. 2, some compounds conferred no antioxidant capacity by themselves, but only after enzymatic reaction and application onto the surface of PS; however, the opposite effect was observed for the TA compound.

Table 2 Chemical structures and abbreviation of the tested compounds.

\begin{tabular}{|c|c|c|c|}
\hline Compound & Abbreviation & Structure type & Structure \\
\hline Caffeic acid & $\mathrm{CA}$ & Cinnamic acid & \\
\hline Para-hydroxybenzoic acid & $\mathrm{pHB}$ & $\begin{array}{l}\text { Hydroxybenzoic } \\
\text { acid }\end{array}$ & \\
\hline Acetosyringone & AS & $\begin{array}{l}\text { Hydroxybenzoic } \\
\text { acid }\end{array}$ & \\
\hline Syringaldehyde & SA & $\begin{array}{l}\text { Hydroxybenzoic } \\
\text { acid }\end{array}$ & \\
\hline p-coumaric acid & pCA & Cinnamic acid & \\
\hline Vanillin & $\mathrm{V}$ & $\begin{array}{l}\text { Hydroxybenzoic } \\
\text { acid }\end{array}$ & \\
\hline Acetovanillone & AV & $\begin{array}{l}\text { Hydroxybenzoic } \\
\text { acid }\end{array}$ & \\
\hline
\end{tabular}




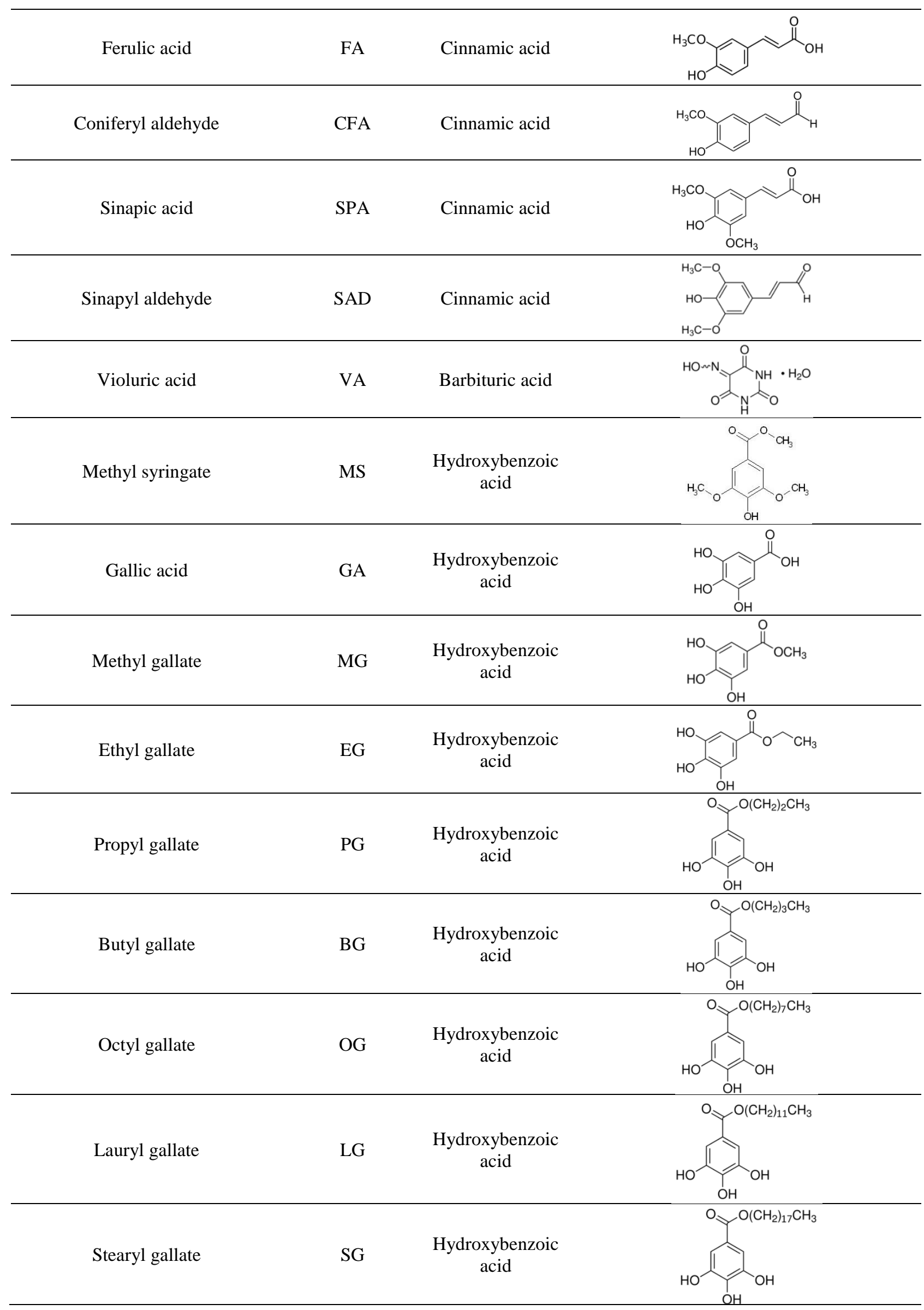




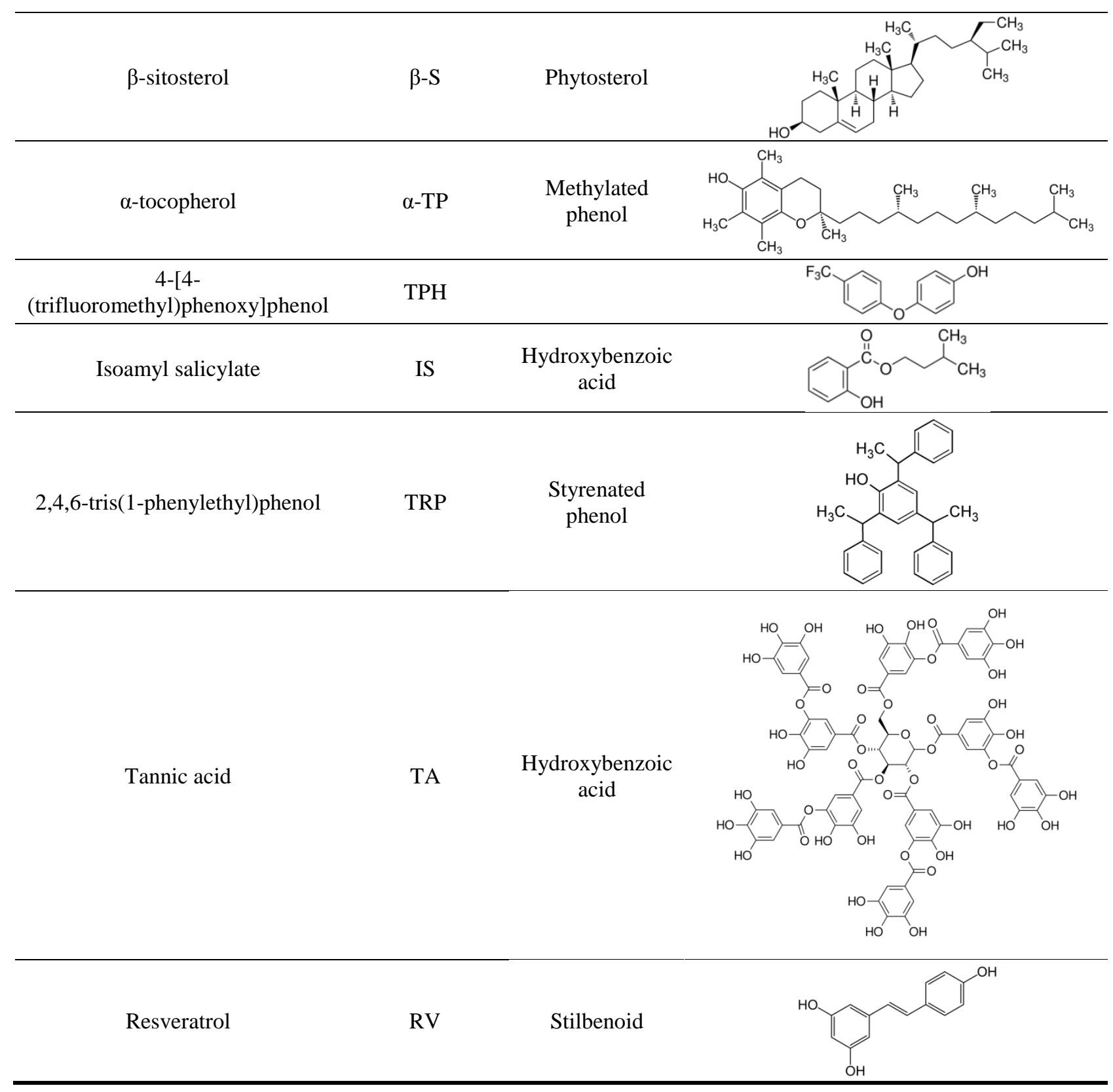




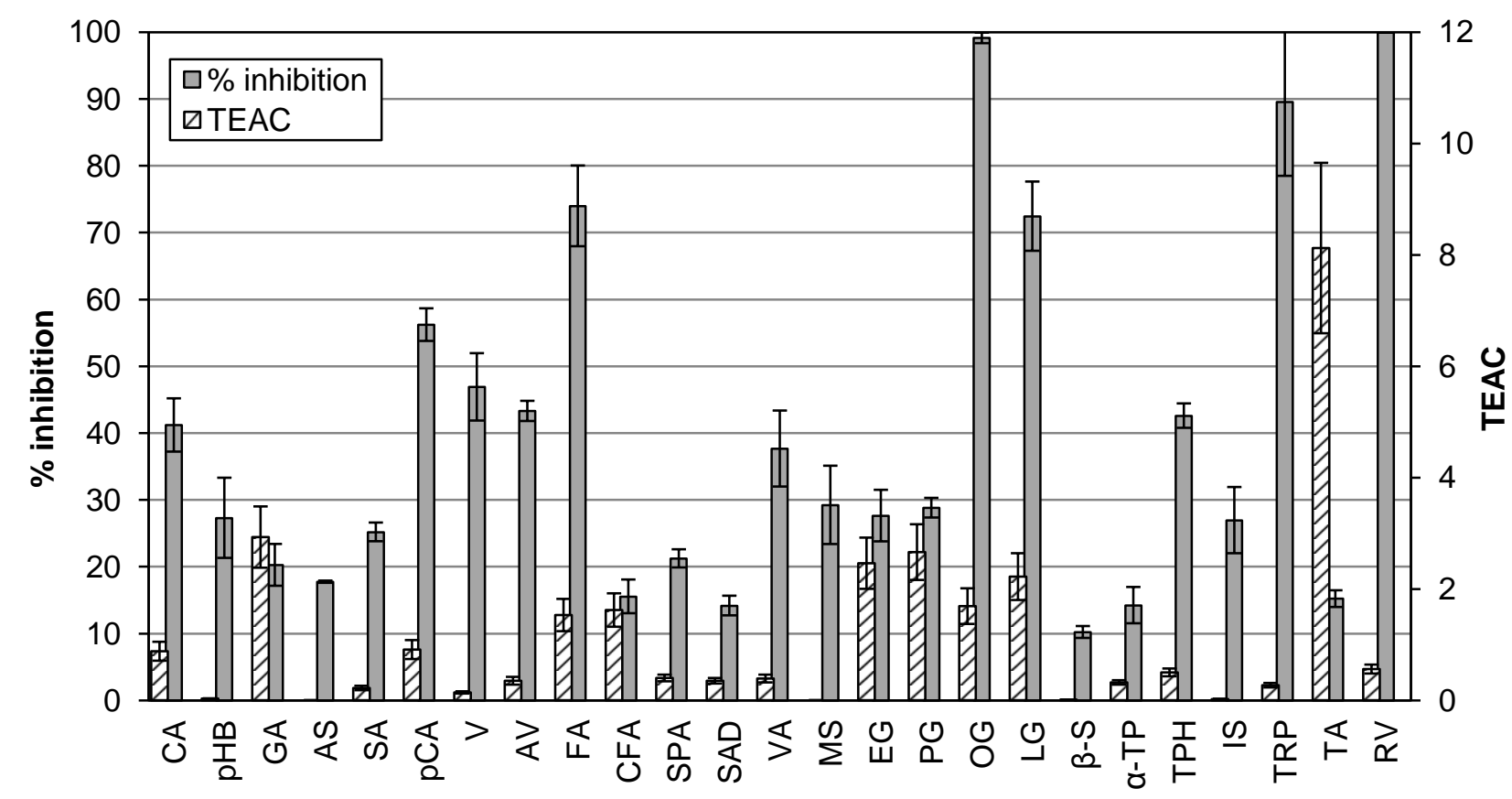

Fig. 2 TEAC values for the pure compounds and antioxidant capacity of PS after surface treatment with each of the 25 functionalization solutions obtained by reaction with Laccase. Antioxidant capacity is expressed as percent inhibition.

The relationship between the $-\mathrm{OH}$ functions in the aromatic ring and the antioxidant capacity appeared not so clear once the phenolic compounds were treated with the laccase. In general, the results suggest that the laccase induces a modification to the structure of the phenolic compound, so that it provides antioxidant capacity when applied onto the cellulosic substrate.

In order to examine the potential relationship between the TEAC for the pure compounds and the final antioxidant capacity conferred to PS by using the enzymatic functionalization solution, we selected the phenolic compounds with the highest antioxidant capacity upon treatment of the PS, namely: FA, OG, LG, TRP, RV and pCA. Antioxidant capacity, expressed as percent inhibition, was measured after washing the treated PS samples. Fig. 3 shows the selected compounds in increasing sequence of TEAC; as can be seen, LG exhibited the highest value and TRP the lowest.

There was no clear-cut relationship between TEAC for a given compound and its ability to develop antioxidant capacity in PS upon enzymatic modification and application under the form of a functionalization solution to PS. However, a slight tendency of antioxidant capacity to increase with increasing TEAC was observed. The PS samples treated with solutions of the pure, enzymatically untreated compounds exhibited no such tendency, so their low inhibition values can be ascribed to the loss of phenolic product upon washing of the substrate. No such loss was observed -inhibition remained high - from PS treated with enzymatically modified compounds. These results suggest the favourable effect of the enzyme, which alters the chemical structure of the phenolic compounds and facilitates their anchoring to cellulose fibres as a result. 


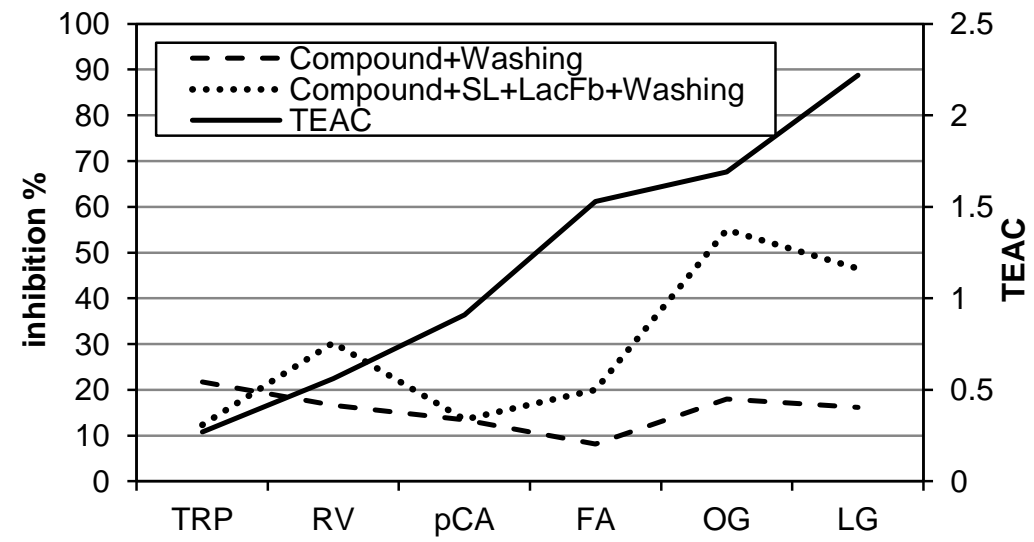

Fig. 3 TEAC values for the pure compounds (solid lines), and percent inhibition of PS impregnated with the pure compounds (dashed lines) and with laccase-treated compounds (compound $+\mathrm{SL}+\mathrm{LaccFb}$, dotted lines), after washing. The studied compounds were FA, OG, LG, TRP, RV and pCA.

The chemical structure of the phenolic compounds may play a prominent role in the intended correlation. Since in the present section only six compounds were analyzed, the study could be extended to additional compounds exhibiting also high correlation. Then, it would be possible to establish a relationship given by several compounds with increasing antioxidant capacity degrees (assessed by the TEAC). This relationship would be useful to predict the antioxidant capacity of the FS-treated PS by only measuring the TEAC value of the compound, without the need to launch the enzymatic reaction and PS treatment. Such a curve might also be useful to ascertain whether antioxidant capacity depends on the chemical structure of the particular phenolic compound.

One can therefore use a suitable phenolic compound to prepare an effective post-enzymatic solution for efficiently functionalizing the surface of cellulosic substrates. In any case, we judged it essential to examine the influence of the enzyme type, strength of grafting, and paper type in order to more accurately assess antioxidant capacity.

The following sections describe the experiments used to these ends. The compounds, enzymes and paper substrates used in each study were chosen in accordance with the particular target parameter.

\section{The relevant effect of the enzyme on antioxidant capacity and bonding strength of FS}

The effect of the enzyme on the development of antioxidant capacity with FS was assessed by using two of the compounds previously found to result in high, similar inhibition, namely: ferulic acid (FA) and lauryl gallate (LG). Both also had a similar TEAC value (Fig. 2). Tests were conducted on eucalyptus PS, using laccase from Trametes Villosa (Tv). In order to evaluate the bonding strength of FS to the cellulosic material, antioxidant capacity was also measured in treated PS after being subjected to heavy washing with hot water at $80^{\circ} \mathrm{C}$ for $30 \mathrm{~min}$.

Fig. 4 illustrates the antioxidant capacity, as percent inhibition, of paper sheets treated with functionalization solutions containing FA or LG in pure or enzymatically modified form. The control 
solutions (KFS) consisting on pure FA and LG were obtained identically as the FS, but using no enzyme. As can be seen, the enzymatically-modified ferulic acid and lauryl gallate (LacTv+FA and LacTv+LG), increased the antioxidant capacity of PS to some extent. This result justified using the enzyme to develop antioxidant capacity.
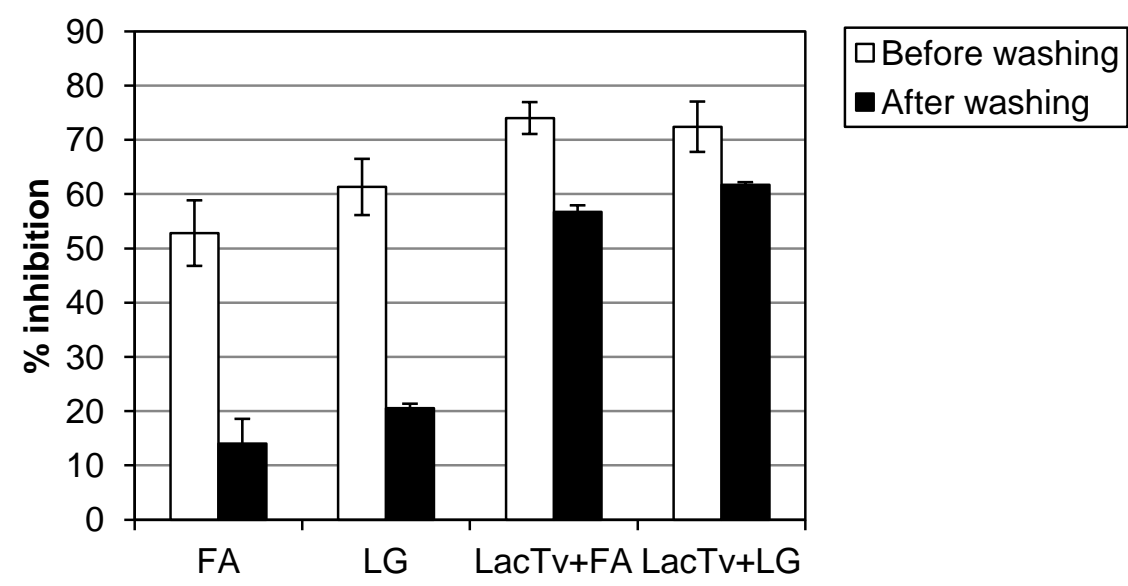

Fig. 4 Antioxidant capacity of the PS after surface treatment with enzyme-modified and unmodified FA and LG, before and after heavy washing.

In addition, the antioxidant capacity of pure (enzymatically unmodified) FA and LG was dramatically reduced by the washing treatment in relation to their enzymatically modified counterparts. Thus, antioxidant capacity remained high upon washing of the substrates treated with the enzymatically modified compounds. This provides an indication of the extent to which the antioxidant chemicals remain attached to the cellulosic material after washing and is very important in relation to the potential migration of chemicals from food packaging. Food packaging can in fact be a source of chemical food contaminants and increasing concern with risk of chemicals migrating into packaged foods has raised the demand for low-migration materials in Europe. These results show that the chemical modification introduced by the enzyme not only boosts antioxidant capacity, but also increases the bonding strength of the antioxidants to cellulosic materials.

In order to elucidate the effect of the enzyme type on the development of antioxidant capacity in PS the LG compound was treated by using three different laccases, namely: a laccase from Trametes Villosa (Tv), a laccase from Cerrena Unicolor $(\mathrm{Fb})$ and a laccase from Myceliphthora Thermophila (Mt). The enzymes were used to obtain three different FS that were subsequently applied to filter PS prior to measuring antioxidant capacity.

As can be seen from Fig. 5, antioxidant capacity was markedly dependent on the particular enzyme used. Based on the results, the best enzyme candidate was $\mathrm{Tv}$, followed by $\mathrm{Fb}$ and Mt. Results demonstrated that the antioxidant capacity can be increased by up to $30 \%$ ( $\mathrm{Tv}$ with respect to $\mathrm{Mt}$ ) depending on the nature of the enzyme. The results also suggest that the different enzymes may have caused different chemical changes in LG, thereby producing LG-modified molecules differing in antioxidant properties. 


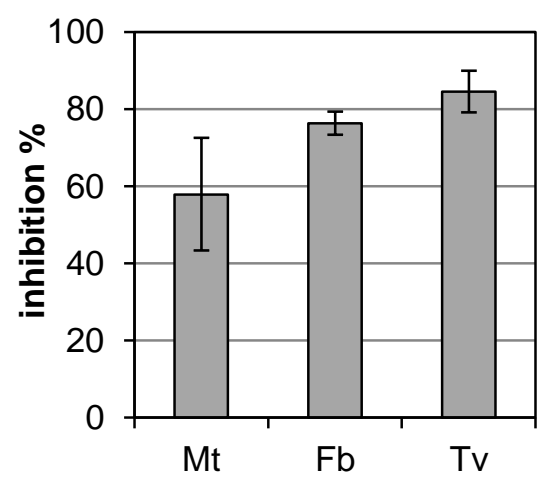

Fig. 5 Antioxidant capacity of the filter PS after surface treatment with the enzymemodified LG compound solution obtained by using three different enzymes, namely: $\mathrm{Mt}, \mathrm{Fb}$ and $\mathrm{Tv}$ enzymes.

\section{Effect of the addition of SL on antioxidant capacity}

In the work described in previous publications (Cusola et al. 2013; Cusola et al. 2014), we used a surfactant (lignosulfonate, SL) to prepare the functionalization solution (FS) for hydrophobizing cellulosic substrates. In our first work, we identified three favorable effects of the presence of SL during the enzymatic reaction, acting as: i) dispersing agent, ii) hydrophobicity enhancer, and iii) enzyme activity preserver. In the second work we observed that the presence of SL during the reaction increased dispersion stability, and reduced the particle size in the case of LG compound; we therefore thought it necessary to also elucidate the potential role of this compound in the development of the antioxidant capacity.

The study described in the present section was aimed at clarifying the effect of this additive on antioxidant activity in PS treated with the proposed methodology. For this purpose, we selected the six compounds exhibiting the highest antioxidant capacity (viz. FA, LG, OG, TRP, RV and pCA) and examined the effect of adding SL. The study was conducted with filter PS and the enzyme Fb. Fig. 6 illustrates the antioxidant capacity of PS treated with solutions of the pure compounds, and the effect of including SL and the enzyme in the formulation of FS; also, it shows the antioxidant capacity of all samples after heavy washing.

The control tests used to assess antioxidant capacity in filter PS samples, both untreated and impregnated with a LacFb solution, provided $6 \pm 1 \%$ and $9 \pm 1 \%$ inhibition, respectively; therefore, neither the paper nor the enzyme by itself was able to develop antioxidant capacity. The dramatic decrease in antioxidant capacity apparent from the black and horizontal-dashed bars in Fig. 6 indicates that the phenolic compounds were weakly adsorbed onto the PS surface and thus easily removed from it by washing. The antioxidant capacity of PS treated with functionalization solutions containing the phenolic compound, SL, and the enzyme ( $\mathrm{LacFb}$ ) (inclinated-dashed bars in Fig. 6) was lower than that obtained with the pure compounds (black bars in Fig. 6). Washing reduced the antioxidant capacity of PS treated with the compound+SL+LacFb solution (grey bars in Fig. 6); however, with LG, OG and RV compounds the antioxidant capacity remained high after washing — which suggests stronger binding of the phenolic compounds to the paper substrate by effect of the action of the 
enzyme on the compounds. Using SL had a favourable effect on the development of antioxidant capacity in PS treated with the proposed method; the effect, however, was dependent on the particular phenolic compound. In fact, the antioxidant capacity of a mixture of phenolic compounds was previously found to be smaller than the sum of the antioxidant capacity of each individual compound to an extent depending on the particular $\mathrm{PhC}$ and its microenvironment. Interactions between compounds can have inhibitory or synergistic effects (Arts et al. 2001; Kuskoski et al. 2005; Blauz et al. 2008). The presence of Lacc in the treatment solution decreased the initial antioxidant capacity of the phenolic compounds, but had a favourable overall effect since it seemingly led to stronger binding of the compounds to cellulosic substrates as shown by the results for paper samples heavily washed with hot water.

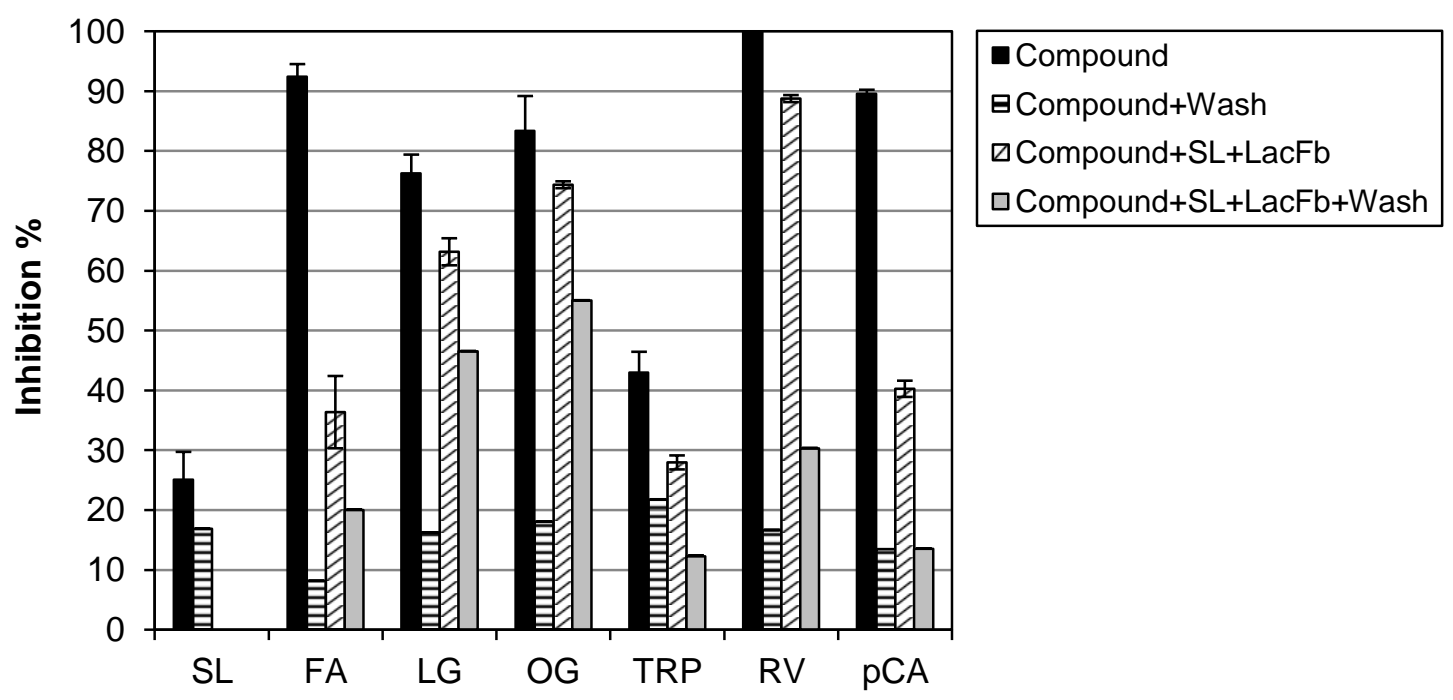

Fig. 6 Antioxidant capacity of filter PS after surface treatment with solutions of the pure compounds alone and in combination with SL+Lacc before and after heavy washing with hot water $\left(80^{\circ} \mathrm{C}\right)$ for $30 \mathrm{~min}$.

\section{Antioxidant capacity of the Lacc-SL-LG (FS) system}

Lauryl gallate is known to be an antioxidant additive used to prevent degradation of food (Aruoma et al. 1993; Kubo et al. 2002). In the present work, it has been proved that it is possible to confer antioxidant properties to paper-based materials by the surface treatment using an FS, prepared from LG compound; in addition, the antioxidant values obtained were significantly high (Fig. 6).

Previous studies showed LG to be effective in hydrophobizing cellulosic substrates by effect of a surface enzymatic treatment, and the effect to be enhanced by the action of the natural surfactant sulfonated lignin improving distribution, hydrophobicity and enzyme activity stability (Cusola et al. 2013; Cusola et al. 2014). Also, as stated above, impregnating PS with the enzymatic product of the reaction of laccase with LG conferred them antioxidant capacity. The study described in this section was aimed at characterizing antioxidant capacity in the functionalization solution (FS) and elucidating the role of its individual components in the development of this property. 
The antioxidant capacity of the LG, SL, LG+SL, SL+Lacc, LG+Lacc controls, and that of the whole treatment (FS), was assessed by the methodology described in the experimental section which is used to achieve the antioxidant capacity of the functionalization solutions (FS), and the pure phenolic compounds. Solutions of the mentioned controls and FS at concentrations ranging from 0 to $2.5 \mathrm{mM}$ were used to measure antioxidant capacity as a function of concentration (see Fig. 7), and the slopes of the resulting curves being compared with that for trolox to calculate TEAC (Table 3). Trolox equivalency is widely used as a benchmark for antioxidant capacity; a TEAC value higher than unity is indicative of a high antioxidant capacity.

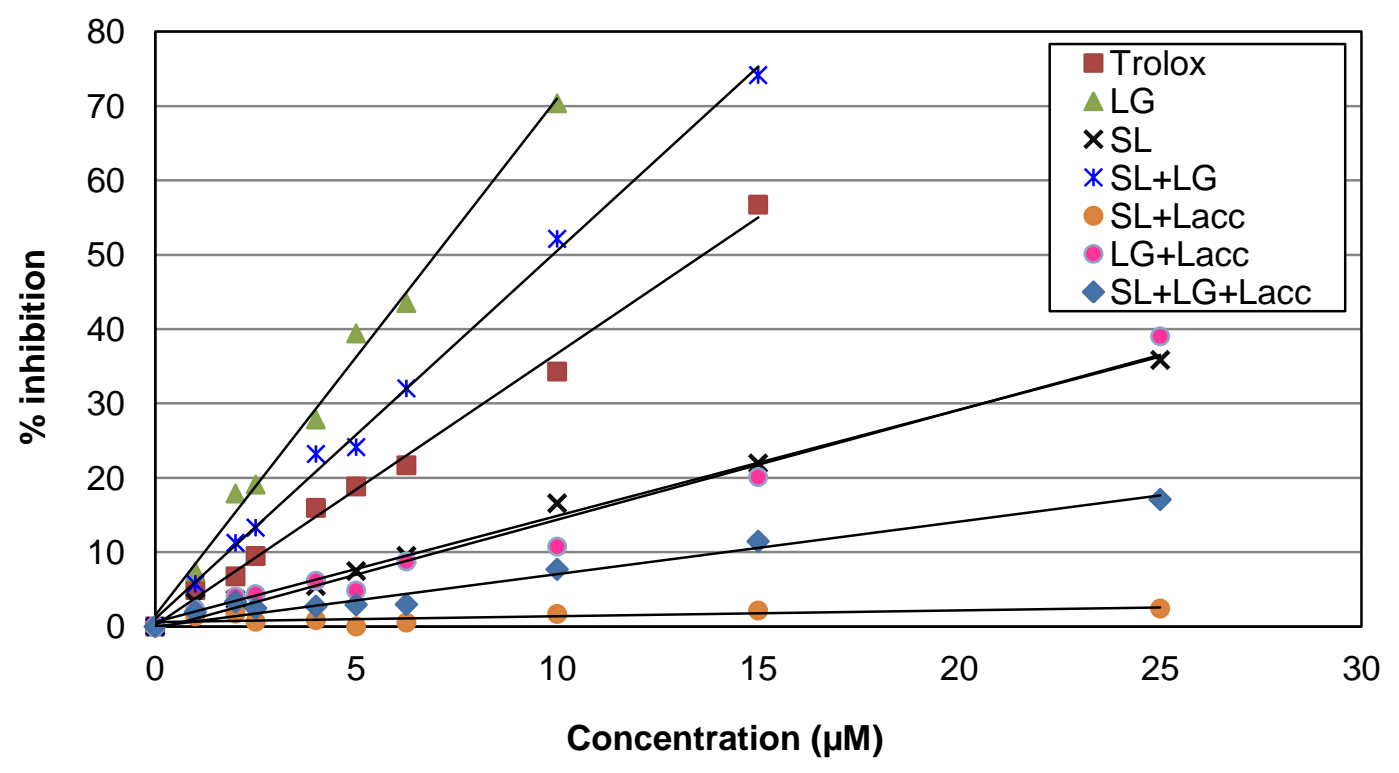

Fig. 7 Variation of inhibition with the concentration of Trolox (reference), LG, SL, LG+SL, SL+Lacc, LG+Lacc and FS.

Table 3 Slopes of the curves obtained from the representation of the percent inhibition versus concentration (Fig. 7), and TEAC values of Trolox, FS, and control solutions.

\begin{tabular}{lcc}
\hline System & Slope & TEAC \\
\hline Trolox & 3.67 & - \\
LG & 7.19 & 1.96 \\
SL & 1.47 & 0.4 \\
LG+SL & 5.07 & 1.38 \\
LG+Lacc & 1.45 & 0.39 \\
SL+Lacc & 0.11 & 0.03 \\
FS (LG+SL+Lacc) & 0.71 & 0.19 \\
\hline
\end{tabular}


As can be seen from the results, the LG and LG+SL controls were those exhibiting the highest TEAC values (1.96 and 1.38, respectively) and hence the highest antioxidant capacity. However, these controls consisted of unmodified LG and SL, so TEAC was considerably decreased upon treatment with the enzyme: to 0.39 with the LG+Lacc control and 0.19 with the functionalization solution (LG+SL+Lacc). This result is suggestive of changes in the phenolic compound (LG) upon enzymatic reaction altering its chemical structure and hence its antioxidant capacity. The TEAC value for the SL and SL+Lacc controls ( 0.4 and 0.03 , respectively) are suggestive of poor antioxidant capacity in SL and even poorer after the enzymatic reaction.

Based on the results, the LG+Lacc and LG+SL+Lacc (FS) enzymatic products, which consisted in aqueous solutions obtained by enzymatic reaction with laccase, possessed no antioxidant capacity. These results contradict those previously obtained for PS treated with these solutions. In fact, as shown in the previous sections, the PS samples subjected to the LG+Lacc treatment exhibited marked antioxidant capacity. There are at least two plausible explanations for this phenomenon, namely:

a) Although the LG+Lacc system has no antioxidant capacity, interacting with cellulose in PS may cause chemical rearrangement of the LG molecule by effect of its grafting onto cellulose and lead to the formation of new structures with antioxidant properties.

b) Although the slope of the inhibition curves for LG+Lacc and FS were very low, the concentrations actually deposited onto the treated PS samples were unknown. In fact, the antioxidant capacity of PS was measured by using a certain amount of paper (10 $\mathrm{mg}$ ), but the exact amounts of LG+Lacc and FS attached to the PS surface could not be determined.

The likelihood of the former hypothesis can be judged partly from the discussion of potential reaction products and grafting adducts. That of the latter requires at least a rough estimate of the amounts of FS products - and hence of modified LG - deposited onto the paper sheets by the treatment. As can be seen in Fig. 2, the samples treated with the KFS consisting in a LG+Lacc solution exhibited an antioxidant value of $73 \pm 5 \%$ inhibition, whereas the antioxidant capacity of PS treated with FS was $74 \pm 5 \%$ inhibition.

Based on the slopes of the curves for LG+Lacc and FS in Fig. 7, obtaining 73\% inhibition required the paper to bear or release amounts of LG+Lacc or FS products in the region of $50 \mu \mathrm{M}$ and $103 \mu \mathrm{M}$, respectively. The amounts of FS the paper was able to absorb after impregnation were evaluated by using a mass-difference method to determine the FS uptake by the paper substrate (commercial filter paper of $62 \mathrm{~g} / \mathrm{m}^{2}$ grammage). The FS uptake (FSU) for the paper was about $68 \mathrm{~g} / \mathrm{m}^{2}$. Since the concentration of LG in FS was $3.55 \mathrm{mmol} / \mathrm{L}$ and the density of FS $1 \mathrm{~g} / \mathrm{mL}$, the amount of FS borne by $1 \mathrm{~g}$ of treated paper was calculated from the following equation:

$$
\left[F S U \times G \times \delta_{F S}\right]
$$

where FSU is the FS uptake, G the paper grammage and $\delta_{\mathrm{FS}}$ the FS density. The amount of FS present in $1 \mathrm{~g}$ of paper was thus calculated to be $1.1 \mathrm{~mL}(\mathrm{FS}) \mathrm{g}_{(\mathrm{paper})}$. Since we used $10 \mathrm{mg}$ of paper to determine the antioxidant capacity of the paper sheets, the concentration of LG in FS allowed us to easily estimate the amount of LG present in each paper sample, which is $0.039 \mu \mathrm{mol}$. Finally, if complete 
release of LG present in the paper into the volume of ABTS solution used $(1.7 \mathrm{~mL})$ is assumed, then the concentration of LG leading to the previously measured inhibition value in treated PS (73\%) was $23 \mu \mathrm{M}$.

Based on the curve of Fig. 7 for FS inhibition, an amount of $23 \mu \mathrm{M}$ of FS could only have caused about $15 \%$ inhibition. Therefore, the amount of LG present in FS - which essentially consisted of enzyme-modified LG molecules - leading 73\% inhibition in treated PS were not able to develop such inhibition percentage when considered in solution without the presence of paper. This result suggests that hypothesis (b) above is not valid and strengthens the likelihood of hypothesis (a).

The previous estimates were obtained on the assumption that FS distributed evenly across PS upon impregnation — which was supported by visual inspection of the PS specimens after impregnation.

\section{Effect of the alkyl chain length of the gallates on the development of antioxidant activity}

According to some authors, hydroxyl groups on the aromatic ring of phenolic compounds are responsible for their antioxidant properties (Robards et al. 1999; Arts et al. 2004; Nugroho Prasetyo et al. 2010). Therefore, the differences in antioxidant properties between unmodified LG and laccasemodified LG described previously must have arisen from chemical changes in LG introduced by the enzyme. In fact, laccase causes chemical alterations in the phenol moiety of LG.

However, as also explained in the previous sections, when enzyme-modified LG interacts with cellulosic materials, chemical re-arrangement of the molecule restores the antioxidant capacity of LG. Thus, the complex formed between enzyme-modified LG and paper possesses antioxidant properties. LG is a phenolic compound belonging to the homologous series of gallates, a series of compounds which antioxidant capacity is widely known. Several gallates are commonly used as food antioxidant additives (Aruoma et al. 1993; Kubo et al. 2002). The major difference between gallates is their variable aliphatic chain length, since they possess an identical core structure (the phenol) and differ only in the length of the hydrocarbon chain.

The present study was undertaken to elucidate the influence of the alkyl chain length on the development of such properties. For this purpose, we selected several compounds from the gallate family. Each gallate was oxidized by the action of laccase and the resulting products, which exhibited liquid properties, were used to impregnate filter PS. Once the treated PS were dry, their antioxidant capacity was assessed. A control consisting of untreated PS samples, one containing the enzyme (viz. paper impregnated with enzyme solution) and one consisting of tannic acid (TA) were also examined for comparison. As can be seen from Fig. 8, the paper itself (without modification) and the laccase control (PS impregnated with the enzyme solution) exhibited very weak inhibition and were thus deemed inactive in terms of antioxidant capacity. 


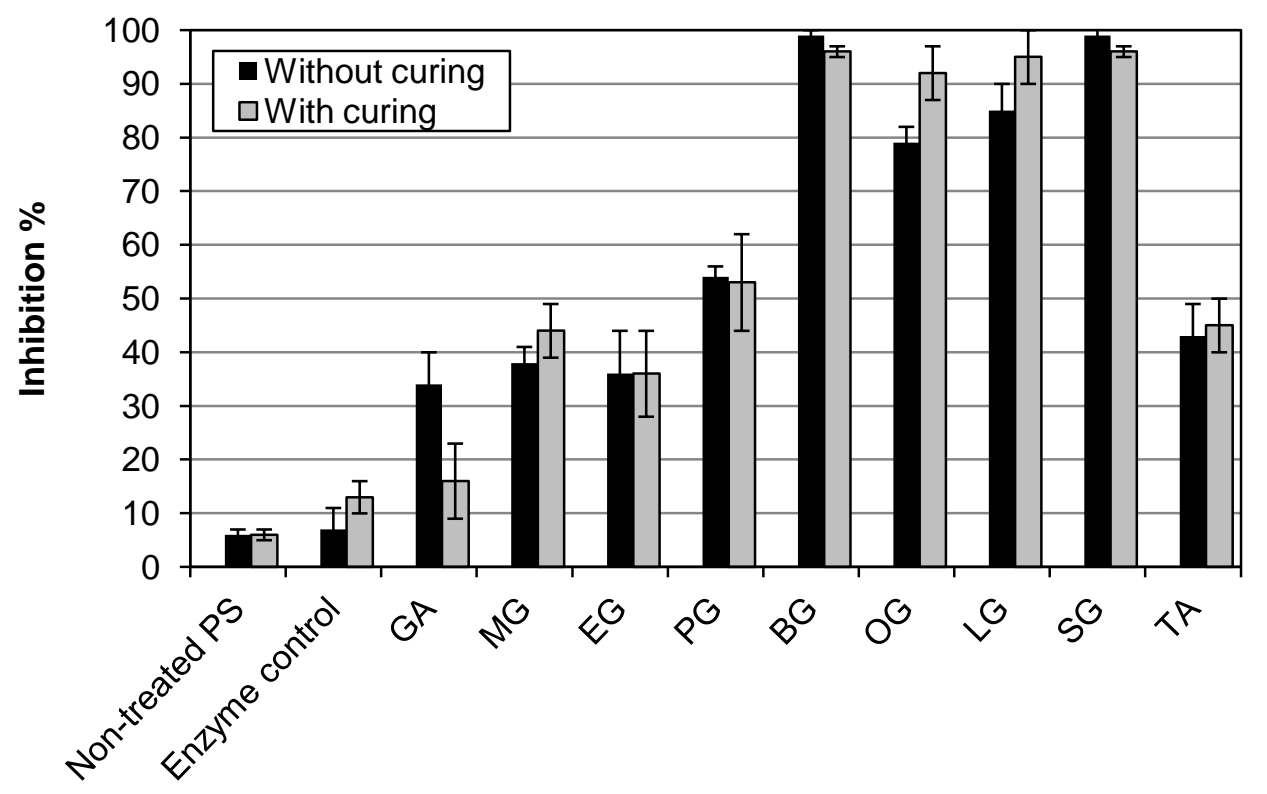

Fig. 8 Antioxidant activity of PS upon surface treatment with each product of the gallate family as prepared by reaction with laccase. The results are expressed as percent inhibition.

Fig. 8 also shows the variation of antioxidant activity with the gallate chain length. Based on the results, the antioxidant capacity of treated PS increased gradually with increasing chain of the gallate but levelled off beyond OG. Since the action of the enzyme focused on the phenol, an identical chemical modification can be expected to have been undergone in all the gallates, and hence no chemical modification in the hydrocarbon chains.

Tannic acid (TA) was also included in the analysis because it contains a large number of phenols and hydroxyl groups potentially influencing the development of antioxidant properties. As can be seen from Fig. 8, TA inhibited oxidation by about $40 \%$. Based on the results, successfully introducing antioxidant capacity with this technique relies more heavily on the presence of a long aliphatic chain in the enzyme-modified compound than on a large number of aromatic rings in its structure.

As stated in previous publications (Cusola et al. 2013; Cusola et al. 2014), heat treatments have a favourable effect on hydrophobicity in treated PS. This led us to also examine the effect of a curing treatment on antioxidant capacity development in treated PS. To this end, PS samples treated with enzyme-modified gallates were oven-cured at $150^{\circ} \mathrm{C}$ for $30 \mathrm{~min}$. Fig. 8 illustrates the effect of the curing treatment; as can be seen, the treatment had no appreciable effect on the antioxidant capacity of PS.

\section{ATR-FTIR analysis of the FS-modified paper and $L G$}

ATR-FTIR analysis was used to find evidences about the grafting of the FS onto the paper sheets. Fig. 9 shows the IR absorbance spectra for the non-treated eucalyptus sheets (Eucalyptus), FS-treated eucalyptus sheets (Eucalyptus+FS), and the lauryl gallate compound (LG). A new peak at $1600 \mathrm{~cm}^{-1}$ appeared in the IR spectrum of the FS-treated eucalyptus sheets with respect the non-treated ones. This 
peak corresponds to the conjugated $\mathrm{C}=\mathrm{C}$ stretching frequency of the aromatic ring in the $\mathrm{LG}$ molecule; the peack is clearly defined when considering the IR curve of the pure LG in Fig. 9. Since the FS contains LG compound in its formulation, the occurrence of this peak at $1600 \mathrm{~cm}^{-1}$ upon the treatment of the sheets using the FS can only be ascribed to the presence of lauryl gallate molecules in the FStreated samples. The absorbance of this peak was weak due to the low concentration of the LG compound onto the paper surface compared to the IR of the pure LG. However, the peak was easily detected.

LG compound was oxidized with laccase under the reaction conditions described in "Materials and Methods" section, albeit in the absence of SL. The product thus obtained, was concentrated and measured by FTIR-ATR. Based on Fig. 10, the hydrocarbon chain of the LG compound remained intact after reaction with the enzyme; in fact, the peaks for methyl $\left(-\mathrm{CH}_{3}, 2850 \mathrm{~cm}^{-1}\right)$ and methylene groups $\left(-\mathrm{CH}_{2}, 2916 \mathrm{~cm}^{-1}\right)$ remained unchanged. This was taken as evidence that enzyme caused no chemical changes in the alkyl chain.

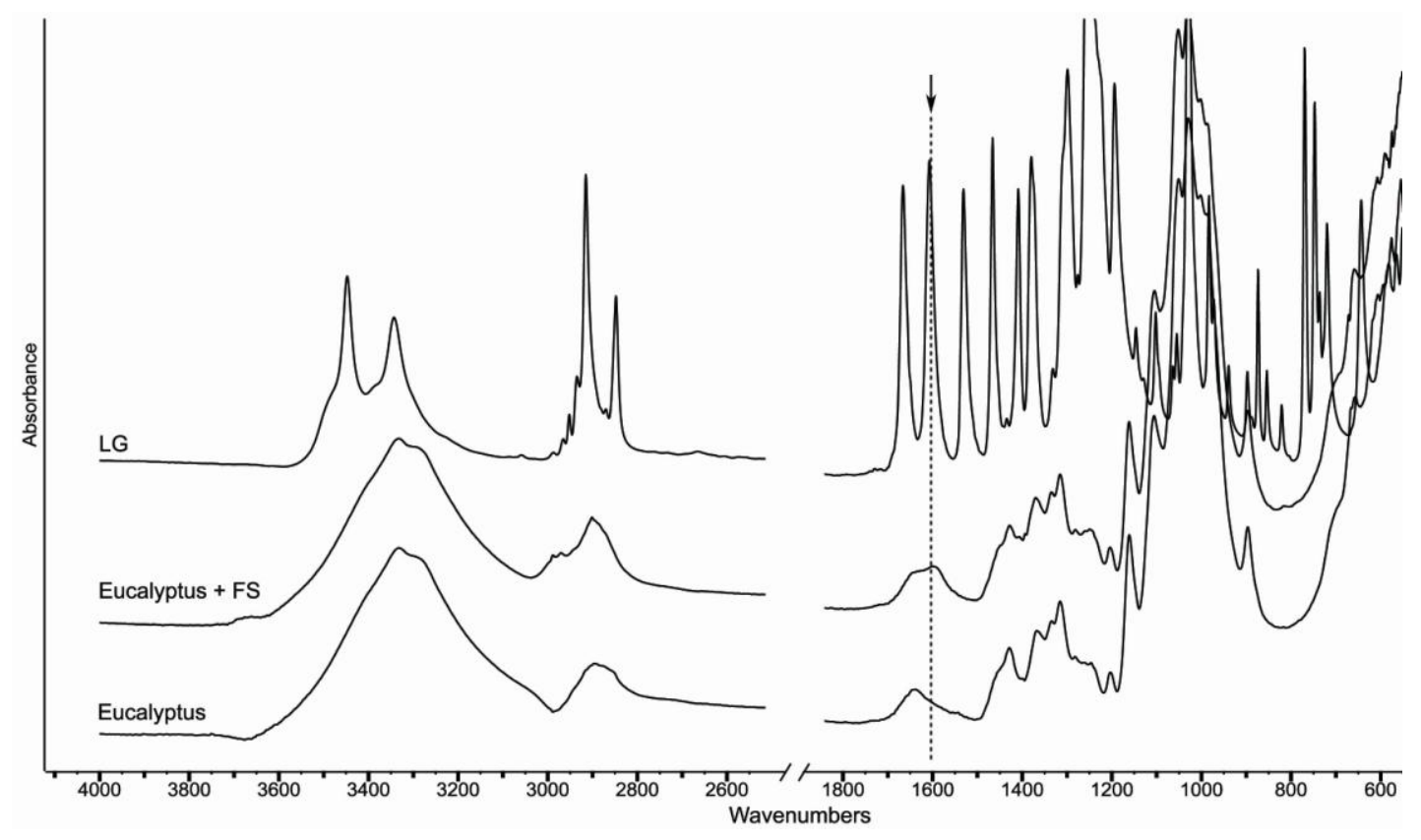

Fig. 9 FTIR spectra of the eucalyptus sheets before and after treatment using the FS, and the pure LG compound. 


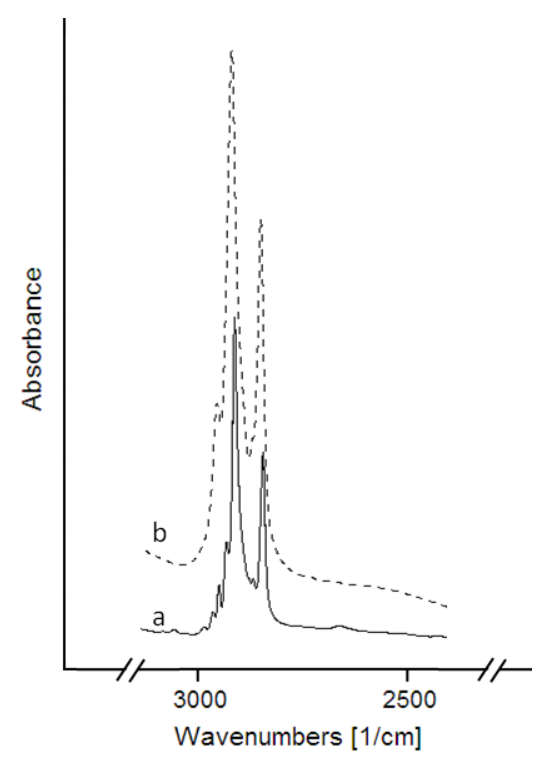

Fig. 10 FTIR spectra for pure (a) and enzyme-modified LG (b) in the range 3100-2500 $\mathrm{cm}^{-1}$.

\section{Conclusions}

A surface treatment of cellulose substrates with the aqueous products derived from the reaction of the enzyme laccase with specific phenolic compounds provides an effective method for developing antioxidant properties in cellulosic materials, after the surface application of such enzymatic solutions. Using the enzyme to introduce chemical changes in the compounds is well justified since, as confirmed by washing tests, it strengthens binding of the modified products to cellulosic materials. The enzyme type used strongly influences the ability of the functionalization solution (FS) to confer antioxidant capacity to paper sheets (PS). The addition of SL to the functionalization solution has a favourable effect on antioxidant capacity development with most phenolic compounds — and an only slightly adverse impact on that of the others. In spite that FS exhibit no antioxidant capacity, it was found to efficiently confer antioxidant capacity after application onto the cellulosic material. Thus, the enzyme causes chemical changes in the LG molecule that reduce its antioxidant capacity, but interaction of enzyme-modified LG with a cellulosic material causes further changes in LG that seemingly restore its antioxidant capacity. The antioxidant capacity of treated PS increased gradually with increasing chain length of the gallate. Therefore, the presence of a long alkyl chain seems to be more important than possessing a large number of aromatic rings here. Antioxidant capacity levelled off in octyl gallate (OG) and was unaffected by a curing treatment. The ATR-FTIR analysis revealed the appearance of a peak at $1600 \mathrm{~cm}^{-1}$ for the FS-treated eucalyptus sheets, providing evidences on the grafting of the FS onto the surface. FTIR analysis also confirmed that the hydrocarbon chain of LG remains unaltered after the enzymatic reaction. 


\section{Acknowledgments}

The authors are especially grateful to the Integrated Project of the Sixth Framework Program BIORENEW (NMP2-CT-2006-026456). The authors also thank the "Ministerio de Economía y Competitividad" of Spain for their support in this work under the projects BIOSURFACEL CTQ201234109 (funding also from the "Fondo Europeo de Desarrollo Regional FEDER) and BIOFIBRECELL (CTQ2010-20238-CO3-01). Special thanks are also due to the consolidated research group AGAUR 2014 SGR 534 at Universitat de Barcelona (UB).

\section{References and Notes}

Aracri E, Fillat A, Colom JF, et al (2010) Enzymatic grafting of simple phenols on flax and sisal pulp fibres using laccases. Bioresour Technol 101:8211-6. doi: 10.1016/j.biortech.2010.05.080

Aracri E, Roncero MB, Vidal T (2011) Studying the effects of laccase-catalysed grafting of ferulic acid on sisal pulp fibers. Bioresour Technol 102:7555-7560.

Aracri E, Vidal T (2012) Enhancing the effectiveness of a laccase-TEMPO treatment has a biorefining effect on sisal cellulose fibres. Cellulose 19:867-877. doi: 10.1007/s10570-012-9686-4

Arts MJTJ, Haenen GRMM, Voss H-P, Bast A (2001) Masking of antioxidant capacity by the interaction of flavonoids with protein. Food Chem Toxicol 39:787-791.

Arts MJTJ, Sebastiaan Dallinga J, Voss H-P, et al (2004) A new approach to assess the total antioxidant capacity using the TEAC assay. Food Chem 88:567-570. doi:

10.1016/j.foodchem.2004.02.008

Aruoma OI, Murcia A, Butler J, Halliwell B (1993) Evaluation of the antioxidant and prooxidant actions of gallic acid and its derivatives. J Agric Food Chem 41:1880-1885. doi: 10.1021/jf00035a014

Barbosa-Pereira L, Cruz JM, Sendón R, et al (2013) Development of antioxidant active films containing tocopherols to extend the shelf life of fish. Food Control 31:236-243.

Blauz A, Pilaszek T, Grzelak A, et al (2008) Interaction between antioxidants in assays of total antioxidant capacity. Food Chem Toxicol 46:2365-2368.

Cusola O, Roncero MB, Valls C, Vidal T (2012) Preparación enzimática acuosa aislada y uso para la funcionalización de la superficie del papel o soportes celulósicos. P201230852:

Cusola O, Valls C, Vidal T, Roncero MB (2013) Application of surface enzyme treatments using laccase and a hydrophobic compound to paper-based media. Bioresour Technol 131:521-526. doi: 10.1016/j.biortech.2012.12.186

Cusola O, Valls C, Vidal T, Roncero MB (2014) Rapid functionalisation of cellulose-based materials using a mixture containing laccase activated lauryl gallate and sulfonated lignin. Holzforsch - Int J Biol Chem Phys Technol Wood 0:1-9. doi: 10.1515/hf-2013-0128 
Fillat A, Gallardo O, Vidal T, et al (2012) Enzymatic grafting of natural phenols to flax fibres: Development of antimicrobial properties. Carbohydr Polym 87:146-152.

Gao Y, Cranston R (2008) Recent advances in antimicrobial treatments of textiles. Text Res J 78:6072.

Gómez-Estaca J, López-de-Dicastillo C, Hernández-Muñoz P, et al (2014) Advances in antioxidant active food packaging. Trends Food Sci Technol 35:42-51. doi: 10.1016/j.tifs.2013.10.008

Hsu CF, Kilmartin PA (2012) Antioxidant capacity of robust polyaniline-ethyl cellulose films. React Funct Polym 72:814-822.

Jansen J, Cassano R, Trombino S, et al (2011) Polymeric membranes with antioxidant activity based on cellulose esters and poly(vinylidene fluoride)/cellulose ester blends. Cellulose 18:359-370. doi: 10.1007/s10570-011-9492-4

Kähkönen MP, Hopia AI, Vuorela HJ, et al (1999) Antioxidant Activity of Plant Extracts Containing Phenolic Compounds. J Agric Food Chem 47:3954-3962. doi: 10.1021/jf9901461

Kanatt SR, Rao MS, Chawla SP, Sharma A (2012) Active chitosan-polyvinyl alcohol films with natural extracts. Food Hydrocoll 29:290-297.

Kodal Coşkun B, Çalikoğlu E, Karagöz Emiroğlu Z, Candoğan K (2014) Antioxidant Active Packaging with Soy Edible Films and Oregano or Thyme Essential Oils for Oxidative Stability of Ground Beef Patties. J Food Qual 37:203-212. doi: 10.1111/jfq.12089

Kubo I, Kinst-Hori I, Kubo Y, et al (2000) Molecular design of antibrowning agents. J Agric Food Chem 48:1393-1399.

Kubo I, Masuoka N, Xiao P, Haraguchi H (2002) Antioxidant Activity of Dodecyl Gallate. J Agric Food Chem 50:3533-3539. doi: 10.1021/jf011250h

Kuskoski EM, Asuero AG, Troncoso AM, et al (2005) Aplicación de diversos métodos químicos para determinar actividad antioxidante en pulpa de frutos. Ciência e Tecnol Aliment 25:726-732.

López de Dicastillo C, Alonso JM, Catalá R, et al (2010) Improving the antioxidant protection of packaged food by incorporating natural flavonoids into ethylene-vinyl alcohol copolymer (EVOH) films. J Agric Food Chem 58:10958-64. doi: 10.1021/jf1022324

López-de-Dicastillo C, Gómez-Estaca J, Catalá R, et al (2012) Active antioxidant packaging films: Development and effect on lipid stability of brined sardines. Food Chem 131:1376-1384. doi: 10.1016/j.foodchem.2011.10.002

Ma X, Yuan C, Liu X (2013) Mechanical, Microstructure and Surface Characterizations of Carbon Fibers Prepared from Cellulose after Liquefying and Curing. Materials (Basel) 7:75-84. doi: $10.3390 / \mathrm{ma} 7010075$

Morgado D, Rodrigues B, Almeida E, et al (2013) Bio-based Films from Linter Cellulose and Its Acetates: Formation and Properties. Materials (Basel) 6:2410-2435. doi: 10.3390/ma6062410 
Nugroho Prasetyo E, Kudanga T, Steiner W, et al (2010) Laccase-generated tetramethoxy azobismethylene quinone (TMAMQ) as a tool for antioxidant activity measurement. Food Chem 118:437-444. doi: 10.1016/j.foodchem.2009.04.102

Qiu X, Hu S (2013) "Smart” Materials Based on Cellulose: A Review of the Preparations, Properties, and Applications. Materials (Basel) 6:738-781. doi: 10.3390/ma6030738

Raheem D (2013) Application of plastics and paper as food packaging materials - An overview. Emirates J Food Agric 25:177-188.

Re R, Pellegrini N, Proteggente A, et al (1999) Antioxidant activity applying an improved ABTS radical cation decolorization assay. Free Radic Biol Med 26:1231-1237. doi: 10.1016/S08915849(98)00315-3

Rice Evans C, Miller N, Paganga G (1997) Antioxidant properties of phenolic compounds. Trends Plant Sci 2:152-159. doi: 10.1016/S1360-1385(97)01018-2

Robards K, Prenzler PD, Tucker G, et al (1999) Phenolic compounds and their role in oxidative processes in fruits. Food Chem 66:401-436. doi: 10.1016/S0308-8146(99)00093-X

Rudra SG, Singh V, Jyoti SD, Shivhare US (2013) Mechanical properties and antimicrobial efficacy of active wrapping paper for primary packaging of fruits. Food Biosci 3:49-58. doi: 10.1016/j.fbio.2013.07.002

Saastamoinen P, Mattinen ML, Hippi U, et al (2012) Laccase aided modification of nanofibrillated cellulose with dodecyl gallate. BioResources 7:5749-5770.

Serpen A, Capuano E, Fogliano V, Gökmen V (2007) A new procedure to measure the antioxidant activity of insoluble food components. J Agric Food Chem 55:7676-7681.

Serrano Cruz MR, Villanueva-Carvajal A, Morales Rosales EJ, et al (2013) Controlled release and antioxidant activity of Roselle (Hibiscus sabdariffa L.) extract encapsulated in mixtures of carboxymethyl cellulose, whey protein, and pectin. LWT - Food Sci Technol 50:554-561.

Shogren RL, Biswas A (2013) Preparation of starch-sodium lignosulfonate graft copolymers via laccase catalysis and characterization of antioxidant activity. Carbohydr Polym 91:581-585. doi: 10.1016/j.carbpol.2012.08.079

Spizzirri UG, Iemma F, Puoci F, et al (2009) Synthesis of antioxidant polymers by grafting of gallic acid and catechin on gelatin. Biomacromolecules 10:1923-30. doi: 10.1021/bm900325t

Tian F, Decker EA, Goddard JM (2012) Development of an iron chelating polyethylene film for active packaging applications. J Agric Food Chem 60:2046-52. doi: 10.1021/jf204585f

Valls C, Roncero MB (2012) Antioxidant property of TCF pulp with a high hexenuronic acid (HexA) content. Holzforschung 67:257. doi: 10.1515/hf-2012-0114 